\title{
Variations
}

Variations

Revue internationale de théorie critique

$19 \mid 2016$

Critique des humanités numériques

\section{À la charnière des milieux académiques et des mobilisations sociales}

Entretien des animateurs du réseau de recherche TERRA avec Alexander Neumann

Marc Bernardot et Jérôme Valluy

\section{OpenEdition}

Journals

Édition électronique

URL : http://journals.openedition.org/variations/768

DOI : 10.4000/variations.768

ISSN : 1968-3960

Éditeur

Les amis de Variations

Référence électronique

Marc Bernardot et Jérôme Valluy, «À la charnière des milieux académiques et des mobilisations

sociales », Variations [En ligne], 19 | 2016, mis en ligne le 06 avril 2016, consulté le 20 avril 2019. URL

http://journals.openedition.org/variations/768; DOI : 10.4000/variations.768

Ce document a été généré automatiquement le 20 avril 2019.

Les ami•e•s de Variations 


\title{
À la charnière des milieux académiques et des mobilisations sociales
}

Entretien des animateurs du réseau de recherche TERRA avec Alexander Neumann

\author{
Marc Bernardot et Jérôme Valluy
}

\section{Alexander Neumann : Comment le réseau de recherche TERRA a-t-il émergé ?}

Jérôme Valluy : Il n'est né ni d'une maison d'édition classique, ni d'une équipe intégrée dans un dispositif universitaire. TERRA est apparu en 2003 comme une modalité de force d'indépendance intellectuelle des enseignants-chercheurs sur le terrain sensible de l'asile, de l'immigration, de la discrimination, du racisme, de l'identité. Nous avons senti qu'il fallait innover pour tenter de trouver un espace de liberté, notamment par rapport à l'agenda scientifique du monde universitaire et pour peser sur ce champ. Dès le début, cela s'est agencé avec le numérique comme moyen de contournement d'un certain nombre d'obstacles d'audience, d'expression ou de collaboration et comme moyen de se faire entendre en poussant la communauté universitaire française à travailler sur l'immigration, la xénophobie, les diverses formes de domination, la construction des frontières, les persécutions des femmes, les camps d'étrangers, et à prendre conscience que ces sujets sont importants mais pourtant très peu traités à cette époque dans l'agenda scientifique. Voilà comment s'est construit le réseau Terra, et l'outil numérique était justement l'instrument principal permettant de s'affranchir des contraintes de marché autant que des contraintes institutionnelles. C'est ce qui a fait que le réseau n'a jamais été institutionnalisé. Nous n'avons pas créé d'association par exemple, le réseau TERRA a toujours été informel, même dans les périodes de forte activité, lorsque nous publions des billets chaque semaine. En revanche, le site s'est doté d'une charte de fonctionnement très détaillée, voire un peu sophistiquée par certains aspects, qui a structuré fortement le réseau et qui résultait d'un travail de négociation de longue haleine dans l'équipe éditoriale. 


\section{A. N. : Vous évitiez ainsi la dispersion qui peut être immanente à la toile ?}

Marc Bernardot : Pas seulement. La charte était aussi le moyen d'assurer l'horizontalité du fonctionnement parce qu'il y avait dans le réseau TERRA, à l'intérieur des différentes équipes qui le constituaient ou qui assistaient aux réunions et aux journées d'étude, aussi bien des universitaires que des militants et des fonctionnaires. Nous avons pris l'habitude de collaborer de manière égalitaire. Il était impossible à long terme de fonctionner sans une structure qui permettrait d'anticiper les conflits, si possible, ou de les régler s'ils apparaissaient. Le fait est que cette charte, qui a mis plusieurs années à émerger et qui existe toujours, est très peu utilisée en réalité, elle nous sert de cadre sécurisant. Ceux qui ont intégré le réseau au fur et à mesure ont trouvé en elle quelque chose d'assez confortable puisque nous n'avons jamais cherché à cliver, à trancher, à voter ou à établir une ligne collective mais au contraire à offrir des espaces de liberté pour la collaboration, l'échange, la publication, les recherches. Cela nous a permis d'avoir un réseau informel mais avec une charte suffisamment solide, presque constituante, assurant une forme de sécurité politique, autrement dit un pluralisme durable.

J. V.: C'est le reflet de nos valeurs démocratiques. C'est une forme de démocratie académique de la recherche, transparente sur la prise de décision par la clarification des positions de l'équipe éditoriale, du comité scientifique, du groupe de modérateurs de la liste "TERRA-Quotidien » et des responsables du support de la revue Asylon (s).Digitales, du «Recueil Alexandries» et des collections. Il y a une répartition des tâches, assez sophistiquée, que la charte, non-juridique mais consensuelle, permet d'assurer sans avoir besoin de chef, de personnalisation, même s'il y a des stakhanovistes, comme Marc et moi, mais qui n'ont jamais désiré être des chefs, ni au sens sartrien ni au sens fonctionnel.

M. B. : D'autant que le réseau a fonctionné par élargissement permanent, les nouveaux venus amenant à leur tour des nouveaux. Nous n'avons pas cherché à concentrer des équipes avec lesquelles nous avions pu collaborer par le passé, à les fusionner, mais au contraire à nous ouvrir à des nouvelles générations de chercheurs et de militants, le plus souvent les deux à la fois, qui travaillaient de manière beaucoup plus spécialisée que nous qui avions été des sortes de pionniers sur un certain nombre de questions dans le domaine, pour résumer cela schématiquement, des sciences sociales des migrations. Nous avons intégré ces nouvelles équipes, formées du fait d'un contexte migratoire porteur en matière de métiers et de sujets et de l'ouverture de l'université à ces questions, apportant des thématiques et des disciplines nouvelles. Cela nous a permis d'élargir les domaines d'étude, depuis les sciences sociales des premières équipes, centrées autour de la sociologie, des sciences politiques, de l'anthropologie, du droit, et de la géographie, jusqu'à une trentaine de disciplines aujourd'hui y compris les arts plastiques, la photographie, la littérature ou la philosophie, qui sont d'autres manières de poser des questions, identiques ou différentes et de formuler des réponses. Les questionnements eux-mêmes se sont élargis aboutissant à des publications sur des thèmes nouveaux. Même à des moments où sa vitalité n'était visible qu'à travers un certain nombre d'outils fonctionnels, le réseau s'est tellement étendu, par le nombre de disciplines ou par sa diffusion internationale, qu'il n'a jamais cessé d'évoluer. Nous n'avons assuré qu'une forme de veille concernant le fonctionnement du réseau, techniquement d'abord, en faisant évoluer les listes et le site par exemple, mais aussi en changeant nos habitudes. Par exemple, nous avons organisé de nombreuses journées 
d'étude les premières années, notamment à la Sorbonne avec Jérôme, ce qui nous donnait une visibilité importante mais demandait une logistique lourde pour la gestion des salles, des listes, des personnes, des thèmes, des invitations, etc. et nous nous sommes lassés. Aujourd'hui, les choses se sont diffractées, beaucoup de journées d'étude pourraient afficher l'étiquette « TERRA » mais nous n'en tenons plus le compte. Certaines manières de travailler se sont estompées, d'autres ont pris la suite, notamment le travail de publication.

\section{A. N. : Où se trouvent donc les lieux fédérateurs de ce développement désormais ? Dans les} comités éditoriaux de revues comme Asylon(s), par exemple?

J. V.: Les lieux fédérateurs ont été nombreux, au sens non matériel du terme. Ils ont d'abord été historiquement ces «Rencontres TERRA » qui avaient lieu tous les mois durant les premières années, d'abord à Paris, pour des questions de facilité, puis dans les établissements des différents collègues de l'équipe éditoriale notamment. Elles ont été très importantes dans la structuration du réseau.

M. B. : D'autant que certaines équipes se sont formées lors de ces rencontres, à partir du vivier de chercheurs des différents domaines présents. À partir d'elles, certains d'entre nous ont monté des équipes de recherche financées par l'ANR, des programmes du CNRS, entre autres.

J. V.: Elles ont aussi été importantes dans la construction de relations entre le monde académique et celui des mouvements sociaux. Dès le départ nous avons pris le parti de réunir des compétences issues de la recherche académique et des associations militant pour le droit d'asile, les sans papiers, etc. Les rencontres étaient l'occasion d'effectuer cette réunion, avec une prise de parole à la tribune souvent partagée entre deux chercheurs et deux activistes ou responsables associatifs avec entre trente et soixantedix personnes à chaque demi-journée...

M. B. : Et jusqu'à deux cents personnes pour les sujets les plus d'actualité et quand les moyens, comme les listes de diffusion, ont fonctionné, dans un contexte où le durcissement déjà présent est devenu particulièrement visible parce qu'il était médiatisé, répété et rhétoriquement marqué en vue de la préparation des échéances électorales des années 2000 qui plaçaient la question des migrations beaucoup plus en avant dans les débats. Nous avons donc bénéficié d'un contexte particulièrement porteur pour ces sujets d'étude jusque dans les années 2008-2009 où les attaques contre l'université ont forcé les équipes à se réorganiser et à utiliser un temps les outils de TERRA pour donner du poids à la mobilisation. Cela a fait rentrer TERRA dans une autre période, avec d'autres thèmes qui n'ont pas été directement éditorialisés mais qui préparaient le passage aux humanités numériques. Nous avions déjà un certain acquis éditorial par des numéros de revue et des publications en accès libre et gratuit, ce qui était la marque propre à l'édition numérique de cette époque, jusqu'à notre rencontre avec les Éditions du Croquant avec lesquelles nous avons édité une vingtaine de livres en moins de dix ans. Mais le mouvement de départ de TERRA a vraiment été cette agglomération de disciplines concernées par les questions migratoires, avant que le réseau ne s'ouvre et ne se généralise avec de nouvelles thématiques plus littéraires, plus artistiques et avec l'intégration de nouvelles questions arrivant au fur et à mesure, comme les problématiques de traduction, les subaltern studies, les postcolonial studies, les queer studies, etc., Le premier livre de TERRA avec les Éditions du Croquant en 2006 concernait par exemple la persécution des femmes. Nous avions déjà construit, avec les 
groupes de chercheurs, avec les témoignages, avec le travail des militants associatifs, la carte de nos futurs élargissements.

\section{A. N. : Comment en êtes-vous arrivés à l'idée d'une collection de livres numériques ?}

J. V. : La dimension d'éditorialisation numérique a été essentielle dès la création de TERRA pour avoir de l'audience et atteindre les différents objectifs que nous avons déjà évoqués, c'est-à-dire peser sur l'agenda scientifique et académique et faire entendre le discours critique des sciences sociales sur des sujets comme l'enfermement des étrangers, les camps d'internement, la xénophobie d'État, la fermeture des frontières, la persécution des femmes, etc. Pour cela le libre accès était fondamental, même si un segment de la production éditoriale était publié aux Éditions du Croquant encore qu'il était parfois simultanément accessible gratuitement sur le site.

A. N. : C'est donc le prolongement d'un mouvement existant plutôt qu'un nouveau projet?

J. V.: Oui. Dans le mouvement actuel du libre accès qui se fonde schématiquement sur la conférence de Berlin de 2002 appelant à la mise en accès libre des publications académiques, TERRA est classé parmi les pionniers par des gens comme Guesdon. Mais nous étions des pionniers tout à fait inconscients de ce qu'ils faisaient. Car nous ne défendions pas le libre accès pour lui-même mais seulement comme moyen de peser sur les débats en étendant notre audience.

Nous bénéficiions d'un certain nombre d'acquis dans les humanités numériques: le principe du libre accès, l'utilisation de l'outil numérique comme moyen de travail dans les sciences humaines et sociales et un réseau entièrement numérique, notre mode de travail le plus fréquent et constant étant constitué de groupes de discussion par mails, des listes de diffusion " TERRA mensuel » ou " TERRA quotidien " pouvant s'élever jusqu'à quatre-vingts ou cent mille destinataires. Nous avons donc, comme M. Jourdain, fait sans le savoir du libre accès et des humanités numériques telles qu'elles se définissent actuellement, pendant des années. Donc, oui, c'est un prolongement mais un prolongement dans lequel nous entendons participer de façon spécifique à la définition de ce que sont, pour nous, les « humanités numériques ».

A. N.: Mais alors qu'apporte la publication de volumes numériques en collection par rapport au fonctionnement de TERRA depuis sa création?

J. V. : La nouveauté vient moins de TERRA que de la question des humanités numériques aujourd'hui. Leur définition n'est absolument pas stabilisée et la conception purement techniciste et instrumentale qui est utilisée ne me convient pas du tout. C'est pourquoi nous parlons d'humanités numériques plurielles, pour appuyer le fait que leur valeur intellectuelle ne peut venir de la simple utilisation des outils numériques dans la recherche en sciences humaines et sociales, mais qu'elle se situe dans leurs rapports avec les différentes valeurs qui traversent les sciences sociales dans une société qui se numérise. Autrement dit, elles doivent porter sur le tournant numérique de la société et les problèmes ou progrès qu'il engendre, par exemple sur les questions du contrôle social, de la transformation des cultures politiques, des formes de mobilisation sociale, des articulations entre la société et l'État, etc. Les humanités numériques plurielles consistent donc moins à se demander ce que l'on peut faire avec le big data, l'analyse d'une masse de données, ou avec les outils numériques, mais plutôt à considérer quel regard critique les sciences sociales peuvent porter sur ce tournant numérique. De la même manière cette critique n'est pas condamnée à une position technophobe de rejet 
de cette transformation de la société mais vise plutôt à réintroduire dans cet objet d'étude des valeurs humanistes, sociales et progressives.

M. B. : Cette nouvelle phase nous permet d'échapper à un certain nombre de problèmes. Nous avons fait le choix, même si nous étions un réseau de publication en libre accès, de diffuser un temps nos publications par le moyen de l'édition classique en collaboration avec les Éditions du Croquant,. L'édition classique présente des avantages, notamment le prestige de l'ouvrage au regard des hiérarchies et des classements, académique et culturelle, qui peuvent exister. Mais ils se sont estompés du fait des effets de la crise générale de l'édition et de la diffusion, plus particulièrement dans le domaine de la critique universitaire touchée par la chute des ventes et la surproduction académique, auxquelles s'ajoutent ceux provoqués par l'affaiblissement du réseau des librairies et de la prise de contrôle de la diffusion par quelques groupes centralisés. Tout cela ne nous satisfaisait pas et faisait que nos livres nous ont semblé avoir de moins en moins d'écho alors qu'ils auraient mérité sans doute une meilleure visibilité, ce que le format papier rendait de moins en moins possible. Il était frustrant de voir les livres être voués au pilon dès leur parution ou presque, alors qu'ils parvenaient à vivre dans leur format numérique. Nous avons donc commencé à travailler à un nouveau modèle il y a trois ou quatre ans, en prenant acte des évolutions du marché et de la concentration des pouvoirs dans l'édition et en cherchant des voies nouvelles que le livre papier ne peut apporter, malgré toute son importance et sa puissance dans notre mémoire émotionnelle et intellectuelle depuis sa constitution moderne en Europe avec Gutenberg. Le livre numérique permet une indépendance et une plasticité accrues, ce qui nous a amenés à concevoir un projet de «livre-portail » qui constituerait une substitution au portail d'entrée classique sur la toile, les gens pouvant accéder gratuitement à un ensemble d'informations organisées par des intellectuels, individuellement ou collectivement, enrichi de documents auxquels ils n'auraient pas techniquement, matériellement ou économiquement accès en format papier.

J. V.: Pour le formuler de façon plus politique, je suis convaincu que le déclin des sciences sociales qui s'atteste dans l'effondrement du marché éditorial et dans la fermeture de départements dans les universités anglaises, suisses, françaises et autres ne vient pas seulement de la transformation idéologique globale néolibérale, sécuritaire et nationaliste mais résulte aussi du fait que depuis dix ans les sciences humaines et sociales ont minimisé le tournant numérique qui est d'une ampleur colossale, et donc n'ont pas su lui faire face. Cela remet en question les modèles économiques, les modes de fonctionnement, les méthodes pédagogiques, etc. La plupart de nos collègues espèrent ne pas voir arriver ces transformations avant leur départ à la retraite pour ne pas avoir à les prendre en charge, ce qui, selon moi, est une grave erreur car c'est l'incapacité des chercheurs en sciences sociales à être présents sur le web et dans la communication numérique qui pénalise fortement leur audience. Le fait que des collègues s'obstinent à enfermer le fruit de deux ou trois ans de recherche dans un livre papier payant qui sera lu par cent personnes est une aberration. C'est une forme d'automutilation des sciences sociales hallucinante car elle n'a plus aucune justification aujourd'hui. La privatisation des résultats de la recherche publique dans un circuit commercial privé avait une justification forte avant Internet, par l'apport considérable pour la démocratie auX $\mathrm{XVIII}^{\mathrm{e}}$, $\mathrm{XIX}^{\mathrm{e}}$ et $\mathrm{XX}^{\mathrm{e}}$ siècles des maisons d'édition indépendantes dans la défense des libertés et l'indépendance de la vie intellectuelle, mais cette justification ne tient plus à une époque où il est possible d'éditer un PDF sur la toile en 
deux clics. J'ai estimé le coût salarial d'un livre sur lequel on travaille à mi-temps pendant deux ans à environ 80000 euros alors que la facture de production éditoriale correspondant aux droits d'auteur est de 8000 euros en fin d'exploitation, c'est-à-dire que l'on prend 80000 euros dans la caisse publique que l'on place à $10 \%$ dans le circuit privé. Si un fonctionnaire faisait de même, on hurlerait au détournement de fonds publics ou à la prévarication. Nous sommes payés pendant deux ans par le contribuable pour une recherche publique censée produire des connaissances aidant la société à s'émanciper mais on mutile l'audience de cette recherche et on la privatise illégitimement, en totale contradiction avec nos valeurs, par tradition et contre la nouvelle donne imposée par le numérique, y compris nos camarades marxistes et défenseurs du service public.

A. N. : Cela me rappelle une idée que j'ai commencé à esquisser lors d'une discussion similaire à l'université de Toronto avec les collègues d'OpenEdition, où mon intervention s'appuyait sur une association entre Johann Gutenberg et la matrice du web : Gutenberg meets Matrix. Une partie de ma famille vient de la région allemande où a eu lieu la révolution protestante, le Palatinat, et qui démarre justement par la circulation du livre, à savoir la Bible à cette époque. Le mouvement protestant et paysan s'appuie sur l'invention technique de Gutenberg pour mener une révolution culturelle. Nous sommes aujourd'hui peut-être dans une problématique comparable. À la mort de Gutenberg en 1468, la technique d'impression du livre n'est absolument pas massifiée mais quelques décennies plus tard se produit la révolution des paysans, à partir de 1525. Luther, Müntzer, tous les théologiens de la libération du XVIe siècle s'appuient alors sur la technique du livre pour rendre accessible l'écrit à un public large qui en était exclu par le monopole du savoir que constituait l'Eglise catholique. Ce monopole est court-circuité par la circulation du livre mais aussi par ce qu'on appelle «l'écrit qui vole», Flugschrift, forme primitive du tract ou de la brochure. Ce sont des formats de circulation du savoir qui ressemblent à ce que vous avez évoqué à l'instant, allant de l'oralité d'une rencontre entre chercheurs, en passant par le compterendu, l'essai écrit, un article de revue ou toute autre publication. Les manuels limitent souvent l'invention de Gutenberg à une histoire de la technique qui se poursuit avec le télégraphe, la radio, etc. jusqu'à Internet, dissociée de la potentialité sociale et de la rencontre avec un public en demande, voire en quête de sens critique dans son époque. Je crois que l'on a eu un exemple de cette relation il y a peu dans les insurrections et occupations de rue de l'Europe du Sud qui se sont emparées de formes de connexion, de circulation d'information et même de mobilisations de savoir, sur le Web et dans la rue ou les conseils de quartier. C'est le cas pour Podemos qui offre plusieurs facettes y compris un courant de sciences politiques critiques qui entre dans sa construction, même s'il y a ensuite des conflits entre l'horizontalité du mouvement et la verticalité d'un parti. Je me demande à quel moment nous entrons dans l'expérience collective d'une rencontre entre une forme de communication et le partage d'un savoir critique qui répond à une demande. Est-ce qu'aujourd'hui, dans un contexte de remise en cause généralisée des institutions françaises, nous sommes dans un moment de rencontre entre la critique et le mouvement?

J. V. : La rencontre n'a pas encore eu lieu, c'est justement tout le problème. C'est la raison pour laquelle nous tournons TERRA vers les humanités numériques, nous voulons dire aux gens, notamment ceux qui font partie de la gauche critique, de ne pas prendre une position de repli face à cette transformation technologique, mais au contraire de s'en emparer pour porter leurs idées, comme ils l'ont fait avant par d'autres moyens. Quelques indicateurs prouvent que cette rencontre n'a pas eu lieu. La présence des sciences humaines et sociales critiques sur Wikipedia, par exemple, est quasiment inexistante, parce que les chercheurs de ces domaines refusent pour le moment d'y contribuer, même s'il y a de nombreuses modalités pratiques pour le faire et même si elle constitue le premier mode d'accès direct à la connaissance, un premier 
repérage d'un domaine de connaissance. De même le copyright qui était une défense du droit d'auteur n'est plus aujourd'hui qu'une défense des rentiers de l'édition puisqu'il vient enrichir leur catalogue rempli de soixante-dix ans de production professionnelle en sciences sociales alors que la rémunération dérisoire de l'auteur lui paie à peine un déjeuner au restaurant. Cela rend les sciences sociales muettes sur la toile, c'est inacceptable d'enfermer ainsi le savoir à une époque où le développement de la bibliographie sur la toile est si important qu'il sature les capacités de lecture des citoyens comme de nos étudiants. Ils continuent à fréquenter les bibliothèques mais ne consultent pas les ouvrages parce qu'ils ont sur leur ordinateur mille fois plus de documents que ce dont ils ont besoin. C'est absurde de continuer à enfermer ainsi la recherche dans le régime du copyright.

M. B. : Durant les premiers temps de TERRA Nous fonctionnions avec ce qu'on appelle le Web.1 c'est-à-dire les listes d'e-mails, les moteurs de recherche, le site, les blogs, etc. Aujourd'hui, c'est-à-dire un peu plus de 10 ans plus tard, nous sommes dans une situation de grande nouveauté. Nous venons de faire la promotion de notre premier livre numérique, dynamique, indépendant et en accès libre, intitulé Actualité de l'habitat temporaire dirigé par une équipe de jeunes chercheurs, notamment Gaëlla Loiseau et Grégoire Cousin. Celui-ci porte sur une question historique de TERRA, l'habitat, en particulier mobile, temporaire, éphémère, etc., d'abord repérée par l'intermédiaire des questions migratoires et de mobilité transnationale, et qui s'est précisée ensuite avec de nouvelles équipes de recherche qui s'en sont emparées. La communication de cette diffusion passe beaucoup moins par les mails désormais que par les réseaux sociaux comme LinkedIn, Académia, ResearchGate, Facebook dont aucun n'a un fonctionnement identique aux autres. Il nous faut donc développer une communication adaptée à chaque réseau qui nous oblige à une mise à jour permanente, qui nous renvoie d'ailleurs à la question de l'évitement de la question numérique, à la fois comme pratique et comme objet, par les sciences sociales, évitement relatif mais massif. Nous sommes dans une situation où nous devons réapprendre à utiliser les outils en permanence, comme un sorte de chantier sans fin où tous les cinq ans ceux que nous maitrisons ne sont plus efficients et ne correspondent plus aux pratiques. Cela me rappelle le travail de $\mathrm{H}$. J. Martin et L. Febvre (1957) sur l'apparition du livre où ils montraient que le passage de la copie du scribe à l'imprimerie dans des universités comme la Sorbonne, s'était fait sans transfert de personnel ou de compétence. Le monde de la copie a survécu quelques temps à l'invention de l'imprimerie par Gutenberg, ou plutôt à sa diffusion en Europe après son invention en Corée deux cents ans auparavant. Or ce ne sont pas les personnels de la copie qui se sont emparés de cette invention nouvelle, mais ceux de la bijouterie capables notamment de gérer le travail minutieux de la fonte. Nous sommes dans une situation comparable, avec une grande partie de nos univers professionnels qui se sont faussement convertis au numérique avec des outils de mauvaise qualité, des connexions aléatoires, du matériel obsolète ou bas de gamme et - une des revendications récurrentes des étudiants à l'université reste l'accès au Wifi! Notre idée est que nous avons véritablement la possibilité de couper les ponts avec l'ancien monde de la production, de la distribution et de la diffusion du livre à travers la notion de « livre-portail ». Nous pourrions contrer cette dispersion subie par le domaine des sciences sociales dans l'océan des informations en proposant des points d'entrée cohérents et organisés par univers intellectuel, thématique ou politique avec une dimension pédagogique puisque les 
outils accompagnant la lecture nous permettraient d'élargir le lectorat et de correspondre aux pratiques contemporaines de la lecture. Les gens doivent pouvoir se connecter facilement et avoir accès à la totalité des données sinon ils n'ont accès qu'à de l'abstract, à de la synthèse, c'est-à-dire à de l'information de seconde main. Nous pensons que ces nouveaux portails peuvent être à terme des concurrents des portails monopolistiques comme Google qui ne sont en réalité qu'une forme de sélection drastique de l'information qui ne donne accès qu'à une infime partie de cette dernière, hiérarchisée selon des règles trop peu transparentes en matière de sélection et d'affichage. Nos livres reviennent donc à une forme d'humanité numérique où la décision n'est plus celle d'un algorithme vaguement organisé mais une forme d'algorithme individuel ou collectif d'êtres humains spécialisés qui produisent une information de qualité, si possible correspondant aux questions actuelles.

J. V.: Il faut distinguer deux formes actuelles d'indexation de la documentation accessible aujourd'hui sur le web: une documentation bibliographique au sens large, c'est-à-dire l'ensemble des formes de communication pertinentes du point de vue des sciences sociales. Elle est océanique, tout le monde s'y noie, non seulement les citoyens, mais aussi les étudiants et les intellectuels. Or les plateformes d'éditorialisation ne résolvent pas les problèmes car il s'y passe la même chose que sur Google depuis 1998, c'est-à-dire que la masse des requêtes génère un tel volume de résultats qu'on ne peut les exploiter. Sur les plateformes d'éditorialisation numérique, le nombre d'articles augmentant, la recherche par mots-clefs aboutit à des milliers de références.

A. N. : Sauf qu'un référencement professionnel et une indexation par mots-clés y existe, qui permet de cerner davantage l'information..

J. V. : Nous faisons de l'indexation par mots-clefs sur TERRA depuis dix ans, mais c'est très intellectuel. Choisir les quelques mots-clefs dans un article réclame de le lire et de le comprendre suffisamment bien pour en retenir les concepts pertinents. Donc, mis à part les chercheurs spécialisés dans le domaine, peu de gens sont capables de le faire et ce ne sont pas ces spécialistes qui le font dans ces systèmes d'indexation, d'où leur faible pertinence. Cela fausse les requêtes concernant des articles entiers qui disparaissent de toute manière dans la masse croissante de résultats sur les bases de données. Face à cela le livre numérique s'impose en substituant à l'indexation automatique une indexation interprétée et produite par les auteurs eux-mêmes. Autrement dit, le livre doit développer une toile vers le web en libre accès de telle sorte que les personnes qui s'intéressent à un sujet pour des raisons professionnelles, personnelles ou de militantisme puissent avoir accès à un vaste stock documentaire choisi intellectuellement par un auteur ou une équipe de recherche spécialisés, ce qu'un algorithme ne peut faire.

A. N. : Cette manière d'interpréter les savoirs disponibles que vous proposez m'évoque I' Encyclopédie des Lumières. Nous ne sommes pas loin de Diderot...

J. V. : Oui, c'était l'ambition de Diderot et des encyclopédistes. Dans l'idée d'un livre, comme Actualité de l'habitat temporaire évoqué par Marc, qui devient un portail sur la toile, il y a indéniablement une dimension encyclopédique, avec cette différence que nous appartenons à une époque où nous sommes conscients d'être obligés de nous spécialiser dans des domaines pour les maîtriser intellectuellement, rationnellement et en matière de compétence. Wikipedia reste dans la perspective de l'Encyclopédie comme forme universelle de tous les savoirs, alors que la recherche doit plutôt aboutir selon moi à des encyclopédies numériques beaucoup plus spécialisées et beaucoup plus 
maîtrisées intellectuellement, par des spécialistes investis depuis des années sur des thématiques et assumant dans le domaine des sciences humaines et sociales la responsabilité morale et intellectuelle de ce qu'ils produisent. Le gros problème de Wikipedia, c'est l'anonymat de l'écriture pour $99 \%$ des contributions.

J. V.: Pour reprendre l'exemple de l'invention de l'imprimerie et ses effets sur la Réforme protestante, il a fallu attendre le $\mathrm{XIX}^{\mathrm{e}}$ siècle pour que le taux d'alphabétisation s'élève considérablement et donc que des effets de l'imprimerie sur la société globale soient effectifs. Là où il a fallu plus de trois siècles à l'imprimerie, il a suffit de trente ans pour que le numérique ait un impact considérable sur la société globale, il y a une vitesse d'accélération colossale, bien supérieure à celle de l'imprimerie.

A. N. : Cependant, il ne faut pas oublier la transmission orale de la part de quelques lettrés vers les milieux paysans allemands, bien avant la Révolution française, où le peuple insurgé la pratique aussi. La société change alors à travers la discussion publique de l'information, à partir de tracts lus par quelques-uns et transmis oralement par la suite. On oublie souvent cette dimension d'oralité qui diffuse des connaissances sans nécessairement passer par l'expérience directe de l'écrit, et qui pourtant existe encore, entre étudiants au café par exemple ou dans certains quartiers. C'est souvent ainsi que se sont propagées les révolutions dans les temps modernes, par exemple dans la révolution russe où l'information et les nouvelles des événements circulaient alors qu'il y avait pourtant un taux d'illettrisme effrayant...

M. B. :L'histoire de TERRA est en partie celle d'une propagation, faite de constructions de réseau, de transmissions et de constitutions orales par l'agglomération, par coalition de gens qui travaillent sur une question sans nécessairement se connaître au départ et qui font une masse de trente, quarante, cinquante chercheurs dont l'oralité est depuis le début une des modalités de travail.

A. N. : Quels sont les modes de financement du réseau TERRA et de la nouvelle maison d'édition numérique?

J. V. : Nous avions parfaitement conscience que si nous voulions que le réseau TERRA atteigne ses objectifs d'indépendance intellectuelle et d'inscription de thèmes jusque-là absents sur l'agenda universitaire, il fallait poser la question de son indépendance financière et de son rapport au financement. Avec la complicité des chercheurs de l'équipe éditoriale, nous avons joué de la situation de TERRA à la charnière des milieux académiques et des mobilisations sociales en cherchant des financements de type ANR mais en faisant en sorte que TERRA ne soit jamais directement concerné par eux. Il s'agissait toujours de financements latéraux, en réalité portés par un des membres de l'équipe éditoriale et qui intégrait TERRA dans son équipe de recherche en refinançant latéralement un certain nombre d'activités de publication du réseau en relation avec la valorisation de sa propre recherche. C'est cette valorisation sur le site qui a permis son propre financement sur le plan logistique et technique.

M. B.: C'est ce qui distingue TERRA d'un fonctionnement associatif ou d'ONG pour lesquelles se pose la question des modes et de la répartition des financements. Le réseau lui n'a pas besoin de source de financement, il est de type gazeux ou chimérique. Lorsqu'il n'y a pas de financement, nous fonctionnons sur des systèmes de collaboration, par le partage du travail comme par exemple pour les listes de diffusion pour lesquelles nous ne jouons qu'un rôle de modérateurs par l'intermédiaire de petites équipes cooptées. La question de la ligne, de la survie du réseau, de la recherche d'un revenu en vue de le consolider ne s'est jamais posée, ce qui nous permet d'avoir aujourd'hui un réseau légitime pour la mutation vers les humanités numériques et 
l'édition numérique, avec des problèmes technologiques complexes, de financement, de travail, de mise au point et d'invention qui n'ont jamais cessé d'être latéraux. Le fonctionnement de TERRA est une sorte de peer to peer. Le modèle économique de la maison d'édition est quant à lui hybride, ce qui permet de résoudre certaines questions de faisabilité. En effet, nous travaillons pour le moment sur des prototypes qui ouvrent de nouvelles formes n'étant pas de simples transpositions du livre de Gutenberg au format numérique, mais un mélange de ce modèle ancien et de nouvelles problématiques éditoriales, ce qui pose plusieurs problèmes du point de vue technologique et logistique devant être financés en amont, puisque le livre doit être accessible en diffusion intégrale et gratuite dès le jour de sa sortie. Nous cherchons à produire des ouvrages sans distribution ni diffusion privées ou institutionnelles et nous avons utilisé les moyens à notre disposition jusqu'alors, c'est-à-dire des subventions, des programmes de recherche dont les chercheurs nous confient une partie, la possibilité d'utiliser des moyens de financement collaboratif du type crowdfunding avec des sommes peu importantes, des cagnottes, notre but étant de maintenir une facilité de financement de projets éditoriaux à faible coût. Travaillant de manière très spécialisée sur les questions de violence de masse, de genre, sur les subalternes, les dominations, la pauvreté, etc. nous ne pouvons ni ne voulons « faire de l'argent », même pour financer notre structure. Il faut donc que nos coûts de sortie soient faibles, ce qui nous assure une indépendance, une flottaison au long cours.

J. V.: Et en même temps, il n'est pas pensable, en raison même des valeurs que nous défendons, que nous exploitions le travail des sous-traitants, des petites-mains, des auto-entrepreneurs. Nous avons des contraintes des deux côtés puisque nous devons apprécier exactement quels sont les temps de travail dans le développement des CMS, des codages sous SPIP. C'est compliqué, à moins de devenir soi-même codeur ou webdesigner, d'évaluer les dépenses et les salaires de manière juste, sans exploiter les gens et sans augmenter problématiquement les coûts. D'autre part nous ne pouvons ni faire commerce du produit de la maison d'édition, ni accepter des financements qui nous mettraient en situation de dépendance institutionnelle ou politique, ce qui implique des systèmes d'hybridation des financements. Nous avons des idées et des hypothèses à partir du travail sur des prototypes mais il va falloir du temps pour parvenir à stabiliser un modèle.

\section{AUTEURS}

\section{MARC BERNARDOT}

Sociologue, Université du Havre

JÉRÔME VALLUY

Politiste, Université, Paris 1 